\title{
内氧化形核反常现象的一种解释 *
}

彭晓(12) 平德海? 李铁藩 ${ }^{(12)}$ 吴维芳 ${ }^{(1)}$

（11)中国科学院金属腐蚀与防护研究所, 金属腐蚀与防护国家重点实验室, 沈阳 110015 ;

(2)中国科学院金属研究所, 固体原子象开放研究实验室, 沈阳 110015)

\section{关键词内篻化 形核 四面体堆垛层错(stacking fault tetrahedron, 简称 SFT)}

\section{Böhm-Kahlweit 形核模型}

以 $\mathrm{Pt}, \mathrm{Ag}$ 等贵金属为基的二元稀固溶合金在一定的 $\mathrm{O}$ 浓度 ( $\mathrm{O}$ 分压)下会发生内氧化. 研究内氧化的形核机制, 有助于理解内氧化析出物对内氧化合金的机械性能, 如硬度、疲劳寿 命、抗拉强度, 以及蠕变、再结晶等行为的影响 ${ }^{[1]}$. 根据内氧化物的析出受扩散控制的 Böhm 和 Kahlweit ${ }^{[2]}$ 提出的经典模型, 如果合金内氧化时, 在已析出内氧化物的 $X^{\prime}$ 处的前方 $X$ 处, $\mathrm{O}$ 和溶质浓度达到形核的过饱和度, 则有新的内氧化物在 $X$ 处析出, $X$ 与 $X^{\prime}$ 间的距离可以表示 为: $\Delta X=X-X^{\prime}$, 它近似等于析出物间的平均距离 (见图 1), 因此, 内氧化层中, 单位体积内 析出物数量 $\left[N_{\mathrm{v}}(x)\right]$ 可以用下式来表示:

$$
N_{\mathrm{v}}(x) \approx \Delta X^{-3}=\left(\frac{X}{\Delta X}\right)^{3} \frac{1}{X^{3}} .
$$

以 $\mathrm{AgCu}$ 二元合金为例, 如果以 $C^{\prime}{ }_{\mathrm{Cu}}, C^{\prime}{ }_{\mathrm{O}}$ 表示 $X^{\prime}$ 处析出物形成时 $\mathrm{Cu}$ 和 $\mathrm{O}$ 的浓度, $C_{\mathrm{Cu}}^{\mathrm{m}}, C_{\mathrm{O}}^{\mathrm{m}}$ 表示内氧化均匀形核的 $X$ 处的 $\mathrm{Cu}$ 和 $\mathrm{O}$ 的浓度, 根据 Böhm-Kahlweit 形核模型, 则有如下的关 系式:

$$
\frac{X}{\triangle X}=\frac{C_{O}}{C_{\mathrm{O}}^{\mathrm{m}}} \frac{C_{\mathrm{Cu}}^{\mathrm{m}}}{C_{\mathrm{Cu}}-C_{\mathrm{Cu}}^{\prime}},
$$

式中 $C_{\mathrm{O}}$ 表示合金表面的 $\mathrm{O}$ 浓度, $C_{\mathrm{Cu}}$ 表示合金氧化时 $\mathrm{Cu}$ 的固溶浓度. 因此, 如果 $C_{O}$ 一定, 那么由这一模型可以预测, 单位体积内的析出物数量, 即 $N_{\mathrm{v}}(x)$ 与析出物形核点离开表面的 距离的立方 $X^{3}$ 成反比. 具体地说, 在内氧化初期, 主要是均匀形核的过程, 大量细小的氧化 物颗粒析出; 随后的内氧化是析出物长大的过程, 单位体积内析出的颗粒数降低但尺寸增大. Böhm 和 Kahlweit ${ }^{[2]}$ 提出的内氧化形核模型符合许多内氧化的实验事实, 并一直被用于解释 许多研究体系的内氧化形核规律 ${ }^{[3 \sim 5]}$.

\section{2 结果与讨论}

在 $850^{\circ} \mathrm{C}$ 于空气中退火 $12 \mathrm{~h}$ 后, 我们发现含 $\mathrm{Cu}$ 原子量百分比为 0.9 的银合金的内氧

1995-11-17 收稿, 1996-01-11 收修改稿

* 国家自然科学基金和国家新材料高技术专家委员会联合资助项目 
化形核规律符合 Böhm-Kahlweit 模型, 但是含 $\mathrm{Cu}$ 原子量百分比为 3.6 的银合金的内氧化颗粒 析出出现了反常现象, 根据内氧化层的析出物形貌的不同, 可以将内氧化层分为 $X_{1}, X_{2}, X_{3} 3$ 个区域 (见图 2). 在 $X_{1}$ 区域内, 内氧化物 (主要是 $\mathrm{CuO}$, 但高分辨电子显微镜( $\mathrm{HREM}$ ) 的观测 结果表明可能有非化学计量配比关系的 $\mathrm{CuO}_{1+}{ }_{x}$ 析出)均匀形核, 在 $X_{2}$ 区域内, 析出物颗粒明 显长大, 在 $X_{3}$ 的区域内, 单位体积内析出物颗粒的密度与内氧化深度的关系不符合等式(1), 因此, 这里用 Böhm-Kahlweit 模型 ${ }^{[2]}$ 就不能解释析出物的形核规律. 为了弄清这一反常现象 产生的原因, 我们进一步用 HREM 对内氧化层的结构进行原子尺度的观测, 发现内氧化层中 存在大量的 SFT (见图 3). 这种 SFT 是由 $\{111\} 4$ 个面和 $1 / 6\langle 110\rangle 6$ 条棱组成的闭合四面体, 属于空位型堆垛层错, 它是通过许多空位聚集形成的 ${ }^{[6]}$. 我们认为, 内氧化时, 因 $\mathrm{Cu}$ 向表面 和内氧化前沿扩散, 引起 “Kirkendall” 空位由内氧化前沿向试样内部或基体中未氧化区域扩 散, 因此, 空位可能在上述区域聚集形成 SFT, SFT 的尺寸与聚集的空位数有关. Zinkle ${ }^{[7]}$ 的 计算表明, 空位以 SFT 的形式存在于 $\mathrm{Ag}$ 基中, 具有最低的能量, 因而最稳定. 合金内氧化过 程中产生大量的 SFT, 会增加 $\mathrm{Cu}, \mathrm{O}$ 的扩散速度, 如果 SFT 出现于图 1 所示 $X^{\prime}$ 和 $X$ 之间的 $X^{\mathrm{s}}$, 那么, SFT 中的 $\mathrm{Cu}$ 和 $\mathrm{O}$ 的浓度极易达到形核临界值, 根据 Böhm-Kahlweit ${ }^{[2]}$ 的形核机制, 析出物将优先在 $X^{s}$ 而不是 $X$ 处形核, 另外, 在 SFT 处形核所需的能量也较低. 因此, 这些 SFT 提供了新的形核点. 使内氧化由 Böhm 和 Kahlweit 所称的均匀形核转变为非均匀形核, 这可能就是上述内氧化层中某些区域单位体积内析出物的颗粒数随内氧化深度的增加反而增 多的直接原因. 当内氧化温度高, $\mathrm{Cu}$ 固溶量大时, $\mathrm{Cu}$ 的扩散增大会促进空位的产生和 SFT 的形成, 因此, 这种反常现象会更明显. 用内氧化合金中产生的 SFT 会增加析出物的不均匀 形核点的观点, 也可以解释 Böhm-Kahlweit ${ }^{[2]}$ 在研究 Ag-原子量百分比为 5.4 的 Cd 合金内氧 化时发现的, 随内氧化深度增加, 氧化物析出数的降低程度比用 Böhm-Kahlweit 模型预测的要 少的实验事实.

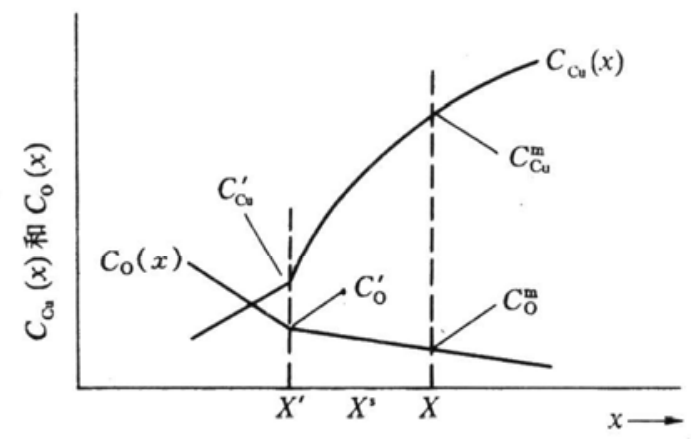

图 1 内氧化方向两个连续的析出物颗粒的形核与溶质 及 $\mathrm{O}$ 浓度的变化关系示意图

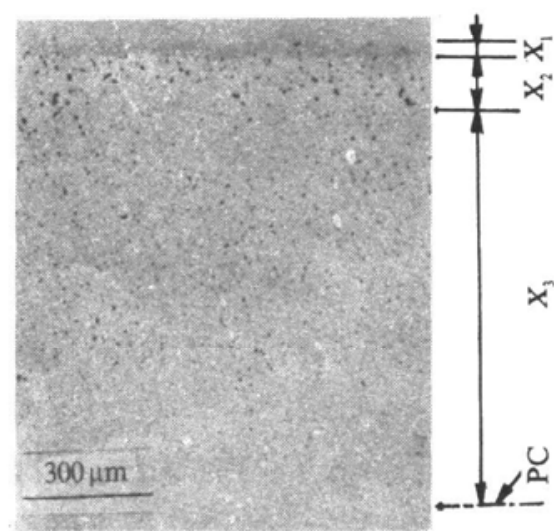

图 2 光学显微镜显示的反常内氧化形貌 $\mathrm{PC}$ 一一样口薄层 


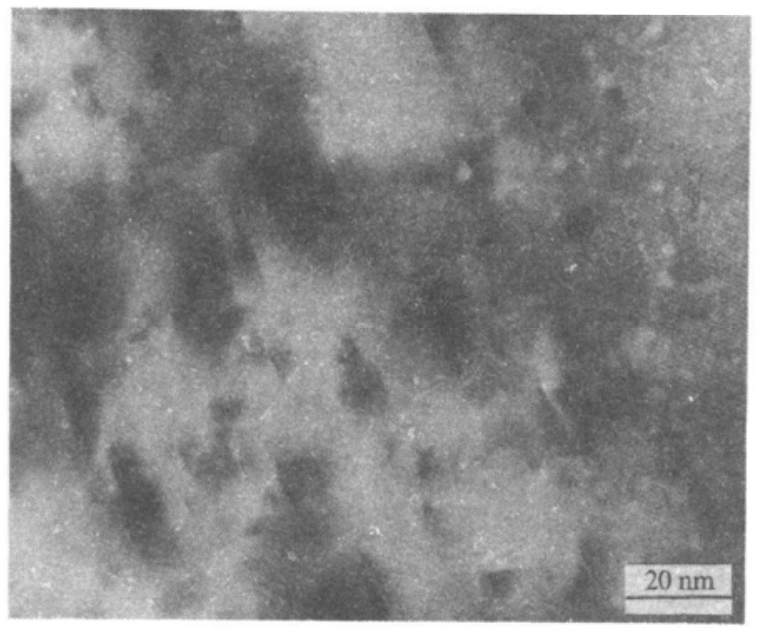

图 3 HREM 显示的内氧化层中形成的 SFT 的原子像

\section{3 结 论}

内氧化 $\mathrm{AgCu}$ 合金的形核反常现象与内氧化层中产生较高密度的 SFT 有关. SFT 会促 进新的析出物的形核而不是已有析出物颗粒的长大.

\section{参考文献}

1 Rapp R A. Kinetics, microstructures and mechanism of internal oxidation, its effect and prevention in high temperature alloy oxidation. Corrosion, 1965, 21:382 401

2 Böhm G, Kahlweit M. Úber die innere oxidation von metallegierungen. Acta Metall, 1964, 12:641 648

3 Whittle D P, Shida Y, Wood G C et al. Enhanced diffusion of oxygen during internal oxidation of nickel-base alloys. Phil Mag A, 1982, 46:931 949

4 Murrle U, Exner H E. Oxide particle densities in internally oxidised Ag-Cd alloys. Mater Sci Technol, 1988, 4:1 141 1 145

5 Douglass D J. A critique of internal oxidation in alloys during the post-Wagner era. Oxid Met, 1995, 44:81 111

6 Sigle W, Jenkins M L, Hutchison J L. Determination of stacking-fault tetrahedra in electron-irradiated silver by high-resolution structural imaging. Phil Mag Let, 1988, 57:267 271

7 Zinkle S J, Seizman L E, Wolfer W G. Stability of vacancy cluster in metals I . energy calculations for pure metals. Phil Mag, 1987, 55A:111 125 